\title{
Programa de salud para la prevención de enfermedades derivadas de la exposición solar en la infancia
}

\author{
BELÉn MORENO GUILLAMÓN \\ al261475@uji.es \\ BELÉN FLOS EXPÓSITO \\ al339176@uji.es \\ ADRIÁN FONTA DE LA FUENTE \\ al339170@uji.es \\ PABlo Salas MEdina \\ psalas@uji.es \\ Águeda Cervera Gasch \\ cerveraa@uji.es
}

\section{Resumen}

Introducción: La evidencia científica respalda que los niños son uno de los grupos de población en los que más debe extremarse la fotoprotección. Una exposición solar irresponsable puede acarrear consecuencias inmediatas como las quemaduras solares. Pero las consecuencias más peligrosas son las que se producen a largo plazo como el fotoenvejecimiento y la fotocarcinogénesis. Por ende, una disminución en la exposición solar en edades tempranas puede disminuir en gran medida la incidencia del melanoma cutáneo en la edad adulta. Por esta razón se realiza un programa de salud con el objetivo de reducir la exposición de rayos ultravioleta (UV) y quemaduras en un $25 \%$ en niños para prevenir una futura aparición de melanomas en edad adulta. Metodología: El programa de salud está dirigido a la población infantil de edades comprendidas entre 6 y 12 años residentes en la Comunidad Valenciana, España (374.555). La captación de los participantes se realizará en las visitas al centro de salud, así como por medio de charlas en los centros docentes. Además, se diseñan trípticos y carteles con el fin de captar la atención de la población. Resultados: Se espera reducir la incidencia de quemaduras en un $25 \%$ en niños entre 6 y 12 años para prevenir futuras apariciones de melanoma en edades adultas en el plazo de 10 años. Discusión: Se considera que la implantación del programa conseguirá que los infantes adquieran hábitos saludables frente a la exposición solar.

Palabras clave: exposición, solar, infancia, prevención, melanoma.

\section{Abstract}

Introduction: The scientific evidence supports that children are one of the population groups in which the photoprotection should be most extreme. Irresponsible sun expo- 
sure can lead to immediate consequences such as sunburn. But the most dangerous consequences are those that occur in the long term such as photoaging and photocarcinogenesis. Thus, a decrease in sun exposure at early ages can greatly decrease the incidence of cutaneous melanoma in adulthood. For this reason, a health program is carried out to reduce the exposure of ultraviolet rays (UV) and burns by $25 \%$ in children to prevent future onset of melanomas in adulthood. Methodology: This health program is aimed at children between 6 and 12 years of age living in the Valencian Community, Spain (374.555). The participation of the participants will be made in the visits to the health center, as well as through talks in the schools. In addition, leaflets and posters are designed to capture the attention of the population. Results: It is expected to reduce the incidence of sunburns to $25 \%$ in children between 6 and 12 years to prevent future occurrences of melanoma in adults ages within the period of 10 years. Discussion: It is considered that the implementation of the program will get that infants acquire healthy habits against exposure to the Sun.

Keywords: exposure, solar, childhood, prevention, melanoma.

\section{Introducción}

La evidencia científica respalda que los niños son uno de los grupos de población en los que más debe extremarse la fotoprotección. En primer lugar, porque los niños son el grupo de población que más tiempo pasa al aire libre. En segundo lugar, porque el principal factor de riesgo de melanoma cutáneo es la radiación ultravioleta que proviene del sol, sobretodo aquella radiación que se produce durante la infancia (Valdevielso-Ramos \& Herranz, 2010). Una exposición solar irresponsable puede acarrear consecuencias inmediatas como las quemaduras solares. Pero las consecuencias más peligrosas son las que se producen a largo plazo como el fotoenvejecimiento y la fotocarcinogénesis, conocida comúnmente como melanoma cutáneo, que es una neoplasia que se origina en los melanocitos de la capa basal de la epidermis, y que se caracteriza por su agresividad y por su alta mortalidad (Lavanderos et al., 2010). La mayoría de los efectos adversos de la exposición al sol son evitables siguiendo unas pautas y utilizando una serie de productos como pueden ser los protectores solares que reducen la incidencia de los rayos UV y consigo los efectos de la radiación sobre la piel. Los protectores solares son sustancias que reducen la incidencia de los rayos UV y consigo los efectos de la radiación sobre la piel. Estos productos se clasifican según el factor de protección solar, que depende de las condiciones del medio y la cantidad de producto aplicado sobre la piel (Martínez et al., 2012). Para considerar que un protector solar es eficaz y proporciona una buena fotoprotección se debe tener en cuenta, en primer lugar, el tipo de filtro solar. Los filtros de los protectores solares se pueden clasificar en: filtros químicos u orgánicos y filtros físicos o inorgánicos. Por una parte, los filtros químicos son sustancias que absorben la radiación ultravioleta y se desencadena una reacción fotoquímica que disminuye los niveles energéticos de dicha radiación tornándose menos dañinos para la piel. Por otra parte, los filtros físicos son sustancias que, por el tamaño y las características de las partículas que lo componen, no dejan pasar la radiación permitiendo así minimizar los efectos de ésta en todas sus longitudes de onda. Los filtros físicos, a diferencia de los químicos, son fotoestables, es decir, no suelen inducir reacciones de hipersensibilidad en la piel por lo que pueden ser usados tanto por adul- 
tos como por niños (Tania et al., 2008). Es importante saber la cantidad correcta de protector solar que se ha de aplicar en la piel ya que de ella depende el factor de protección solar, que nos indica cuánto tiempo más un protector solar aumenta la capacidad de defensa natural de la piel antes de llegar a quemarse (Tania et al., 2008). Por último, hay que tener muy en cuenta la regularidad con la que se usa, así como las re-aplicaciones mientras haya una exposición al sol. Es importante que la protección solar se incorpore a la rutina diaria de la población durante todo el año, especialmente en los niños. Aun así, en niños menores de 6 meses el uso de productos solares se debería evitar ya que podrían ocasionar deficiencias, como por ejemplo la vitamina $D$ que se absorbe gracias a la luz solar, y enfermedades relacionadas con el uso de protectores solares en el bebé (Tania et al., 2008). Por ende, una disminución en la exposición solar en edades tempranas puede disminuir en gran medida la incidencia del melanoma cutáneo en la edad adulta.

\section{Método}

\section{Diseño}

Se diseña un programa de salud, realizado por alumnos de segundo de Enfermería de la Universitat Jaume I en el espacio temporal comprendido desde octubre de 2016 hasta diciembre de 2016. El diseño de este programa de salud se basa en dos pilares, uno de ellos es la formación que recibirán los profesionales sanitarios y escolares, el otro es la captación de los participantes por medio de charlas, trípticos y actividades realizadas en el centro docente.

\section{Población}

El presente programa de salud, dedicado a prevenir la exposición solar, está dirigido a la población infantil de edades comprendidas entre 6 y 12 años residentes en la Comunidad Valenciana, España (374.555). El desarrollo del programa se realizará en las aulas de los centros docentes y en las consultas pediátricas de los centros de atención primaria. En las consultas pediátricas se aprovechará la presencia de los padres, para comentar los aspectos básicos de la protección y proporcionarles un tríptico informativo.

\section{Captación}

Se realizará una captación activa de usuarios aprovechando las visitas de la población al centro de salud para las revisiones pediátricas. Concienciando a la población sobre las consecuencias de una irresponsable exposición solar y la importancia de la protección solar. Por otra parte, durante el curso escolar, se realizarán charlas y actividades periódicas para involucrar en la causa a la población infantil. Además, se intentará recaptar a los usuarios que han participado en el programa pero que han dejado de participar contactando con ellos mediante teléfono o a través de los profesores en los centros escolares. Se diseñarán una serie de carteles y trípticos que serán distribuidos en centros de salud, hospitales y centros docentes con el fin de captar la atención de la población. 


\section{Planificación del programa}

Para la puesta en práctica del programa se deberá planificar tanto la formación de los profesionales como el desarrollo del mismo en los centros de salud y centros docentes.

Planificación de la formación de los profesionales sanitarios:

- Número de sesiones. 5

- Duración de las sesiones. 4 horas

- Lugar. Centro de Salud Pública

- Número de participantes. 20-30

- Anual - entre febrero y marzo

El planteamiento de la ejecución de dicho programa en los centros escolares es:

- Número de sesiones.1

- Duración de las sesiones. 2 horas ( $1^{\circ}$ de primaria) - 3 horas ( $6^{\circ}$ de primaria)

- Periodicidad. Anual - mes de abril ( $1^{\circ}$ y $6^{\circ}$ de primaria)

- Temporalización:

- Tiempo que se destinará a la charla. 45 - 50 minutos.

- Tiempo que se destinará a las actividades. 1 hora ( $1^{\circ}$ primaria) - 2 horas ( $6^{\circ}$ primaria)

- Lugar. Centro escolar (aulas y patio)

- Número de participantes. 20-30

Por lo que respecta al centro de salud, la planificación previa no será igual de necesaria que en los centros escolares. Tanto los pediatras como los enfermeros de pediatría, se encargarán de aprovechar las consultas de los infantes para educarlos en cuanto a exposición solar respecta; para ello los profesionales sanitarios contarán con carteles y trípticos informativos que podrán utilizar como apoyo a la hora de informar a los usuarios.

\section{Recursos necesarios}

En cuanto a recursos humanos, se dispondrá de enfermeros en consultas de pediatría que capten pacientes durante las revisiones del niño sano. Estos enfermeros también realizarán charlas en los centros escolares con el apoyo de los maestros. Además, se contará con pediatras que realicen diagnósticos y, en el caso que fuese necesario, derivasen al dermatólogo para un seguimiento más exhaustivo del caso. Se realizarán cursos de formación de 20h para los profesionales sanitarios que vayan a realizar el programa donde se les educará en la detección rápida de melanomas, así como la forma correcta de realizar los talleres, teniendo en cuenta las edades de los usuarios a los que va dirigido, para llevar a cabo unas actividades u otras. Se contará también, con la presencia de profesionales docentes con el fin de que reciban conocimientos básicos sobre el tema, así como identificar y prevenir su aparición. En cuanto a los recursos materiales se diferenciará entre los recursos existentes y los recursos necesarios para ejecutar el programa de salud. 
Recursos existentes:

- Materiales para el aula: pizarra, proyector

Recursos necesarios:

- Guía para el profesorado

- Manual sobre conceptos básicos para la educación en la prevención del melanoma

- Folletos informativos para padres

- Material para centros sanitarios: carteles y trípticos

- Material para el aula: carteles, fichas de actividades, lápices o rotuladores de colores, tarjetas blancas, pegatinas con los distintos colores de piel, pegatinas de objetos relacionados con la exposición solar, gomas elásticas.

- Material de protección: gorras recortables y muestras de cremas solares de $20 \mathrm{ml}$ con factores de protección solar desde FPS 15 hasta FPS 50.

- Material de evaluación: cuaderno de evaluación de las actividades en el aula.

Para poder contar con todas las facilidades explicadas anteriormente, se necesitarán una serie de recursos económicos. Las ayudas económicas se solicitarán en la Conselleria de Sanitat Universal i Salut Pública.

\section{Actividades para conseguir los objetivos}

Para conseguir los objetivos anteriormente mencionados, así como los resultados esperados, el programa de salud se llevará a la práctica tanto en centros sanitarios como en centros docentes.

\section{Plan de actuación en niños de 6-7 años}

En primer lugar, se realizará una charla educativa donde se explicará cómo actúan los diferentes protectores dependiendo de las características físicas de cada niño, teniendo en cuenta el color de pelo, ojos, piel, etc. La charla consistirá en un relato corto «El Sol y los Ladrillos» que explique de forma didáctica los diferentes fototipos. Una vez finalizada la charla, se procederá a la visualización del vídeo «La Piel». (Érase una vez la vida)» desde el minuto 2:45 hasta el 3:45. El vídeo se encuentra en la plataforma Youtube. En cuanto a las actividades, se les repartirá lápices o rotuladores de colores y diferentes hojas de ejercicios para que asienten los conocimientos que se han explicado en la charla. Además, se darán manualidades adecuadas a su edad. Todo este proceso estará guiado por el profesional sanitario y el maestro.

\section{Plan de actuación en niños de 11-12 años}

En el curso escolar de sexto de primaria se realizará una charla de aproximadamente una hora en la que se introducirán los siguientes conceptos: quemadura, melanoma, protector solar, factor de protección solar, fototipo y escala de Fitzpatrick (Sánchez \& Nova, 2017). En esta charla, además de explicarles el significado de cada uno de los conceptos anteriores se 
les explicará la importancia de tener unos buenos hábitos frente a la exposición solar y cómo pueden adquirirlos.

Una vez realizada la charla se desarrollarán una serie de juegos para que los alumnos puedan aprender de una forma práctica la información que se expone en ella. Esta parte constará de dos juegos. Para introducir el concepto de protección solar, factor de protección y las consecuencias de una exposición solar excesiva como las quemaduras y su relación con la aparición de melanomas, el personal sanitario en conjunto con los docentes del centro realizará un juego didáctico que se denomina «Impacto solar». La actividad se desarrollará en el aula. Para una mejor comprensión y resultado de la manualidad se tendrá que haber introducido previamente el concepto de fototipo y la escala de Fitzpatrick (Sánchez \& Nova, 2017). Por otra parte, se desarrollará la actividad «pasaporte solar» que consiste en que los alumnos realicen una tarjeta identificativa en la que figuren sus datos personales (nombre, apellidos y edad), su fototipo y la protección que más se adecúa a su tipo de piel.

\section{Resultados}

Previamente al diseño del presente programa, se indagó sobre cuál era el problema de salud que más preocupaba a los padres respecto a sus hijos se procedió a la creación de un cuestionario con escala tipo Likert donde quedaban reflejados los problemas de salud más relevantes que pueden presentar los niños en edades comprendidas entre 0 a 18 años (Figura 1). Los resultados obtenidos se analizaron estadísticamente y se observó, que, a diferencia de lo esperado, la exposición solar, que debería ser una de las principales preocupaciones en la zona de la Comunidad Valenciana, por su gran cantidad de horas de sol y por tratarse de una zona costera, es de los supuestos cuyo rango de preocupación es de los más bajos.

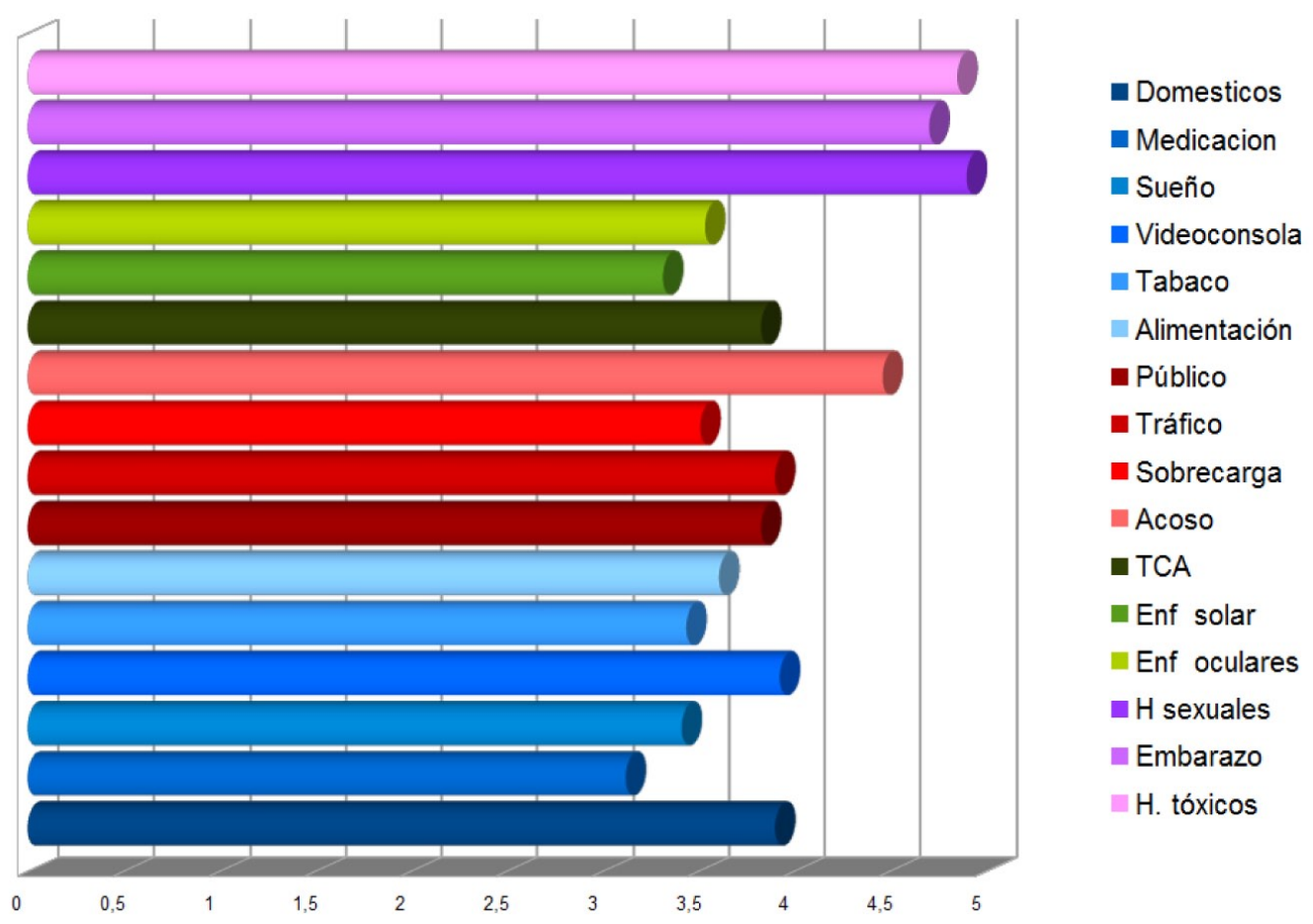

Figura 1. Análisis principales preocupaciones paternales (Elaboración propia) 
Considerando los datos que se obtuvieron, se llegó a la conclusión de que, era de gran importancia desarrollar un programa de salud que abordase la problemática de la exposición solar, así como su prevención durante la infancia.

El programa de salud está dirigido a todos los niños residentes de la Comunidad Valenciana (España) entre los 6 y 12 años, 374.555. Para poder valorar los resultados se deberá conocer aproximadamente cuántos de estos han participado en el mismo por lo que se desarrollará un censo de la población escolar a la que se la realizado la charla. Además, se realizará un recuento de los trípticos informativos que se entregan en las consultas de enfermería. Como último recurso, aunque más utópico se propondría la creación de un apartado específico en el programa Abucasis «asesoramiento prevención exposición solar». Con el fin de poder valorar si los participantes del programa cumplen los nuevos conocimientos adquiridos y los ponen en práctica se les preguntará en cada revisión pediátrica hasta los 14 años (última revisión). Además, se les enviará información en forma de trípticos al comienzo de cada época estival.

Los resultados esperados del programa a largo plazo es el cumplimiento tanto de los objetivos generales como de los específicos. El principal objetivo que se pretendía conseguir con la implantación del presente programa era reducir la incidencia de quemaduras en un 25 $\%$ con el fin de prevenir futuras apariciones de melanoma en edad adulta. Para poder estudiar si ha habido una mejora, se debe conocer la prevalencia de melanoma de la población antes del programa, así como los datos de quemaduras solares recogidas en los niños de 6 y 12 años. En cuanto a las quemaduras, se recogerán los datos obtenidos tras el periodo de un año desde que el programa entre en vigor, y se realizará un seguimiento durante los próximos años con el fin de comprobar si existe una reducción de quemaduras solares y con ello se estén cumpliendo los objetivos marcados. Además, para verificar que la educación para la salud que se ejecutó fue efectiva, se realizará un estudio cada cinco años.

Para comprobar que existe una reducción de la prevalencia del melanoma, se debe dejar un periodo de tiempo más extenso para poder estudiar los datos, puesto que para reducir la prevalencia se debe invertir más tiempo en educación preventiva para poder generar un resultado significativo. Además, en este programa los resultados se valorarán en el momento que los niños que fueron población diana entren en la edad adulta. Por lo tanto, se tendrá que esperar un mínimo de diez años para observar un cambio significativo a nivel evaluable.

\section{Discusión y conclusiones}

La puesta en práctica de un programa de salud desde edades tempranas resulta de gran importancia para promover la salud de los infantes, generando la creación de hábitos saludables en una etapa de vida donde resulta más asequible la adquisición de nuevas costumbres, dada la mayor facilidad de aprendizaje de conductas positivas hacia la salud. (García, 1998). Además, la participación de los centros docentes resulta idónea para la promoción de la salud dado que se puede abarcar una variada población infantil.

Como se ha comentado anteriormente, diversos estudios muestran que una exposición solar irresponsable puede acarrear desde quemaduras hasta consecuencias más peligrosas como el fotoenvejecimiento y la fotocarcinogénesis. (Lavanderos et al., 2010). La decisión de realizar un programa de salud que previene la exposición solar en la infancia fue por dos razones. En primer lugar, porque la Comunidad Valenciana es una zona costera donde las horasde sol abundan y no se cuenta con ningún plan o programa de salud que promocione hábitos saludables frente la exposición solar. En segundo lugar, porque es en los niños donde se 
debe extremar la fotoprotección dado que al pasar más tiempo al aire libre se encuentran más expuestos a la radiación UV (Valdevieso-Ramos \& Herranz, 2010)

Por estas razones, se cree importante, con la aplicación del programa de salud en centros escolares y centros de atención primaria, conseguir que los niños adquieran hábitos saludables en lo que a exposición solar se refiere, puesto que se ha podido comprobar, con el cuestionario sobre los problemas de salud más relevantes en niños de 0 a 18 años, mencionado anteriormente, que este hecho es uno de los que menos preocupa a los padres en la Comunidad Valenciana. Así pues, el único método que utilizan para combatir el problema son los filtros químicos de forma ocasional, siendo estos insuficientes para una adecuada protección solar.

Las últimas revisiones muestran estudios que informan de la existencia de evidencia científica suficiente que asegura que la prevención de melanomas es mayor con el uso diario de filtro solar que con un uso ocasional (Sánchez et al., 2014). Por este motivo se consideramos importante educar a los infantes sobre un buen uso de filtros solares, así como de protección física.

\section{Referencias bibliográficas}

García, I. (1998). Promoción de la salud en el medio escolar. Revista Española de Salud Pública, 72(4), doi: https://scielosp.org/scielo.php?pid=\$1135-57271998000400001\&script=sci_arttext\#ModalArticles

Lavanderos, F., Pérez, P., Jeria, N. \& Concha, D. (2010). Actualizaciones en melanoma maligno cutáneo. Cuadernos De Cirugía, 24(1), 47-56, http://dx.doi.org.10.4206/cuad.cir.2010. v24n1-08

Martínez, M. \& Zúñiga, G. (2012). Los efectos de la luz ultravioleta. La Ciencia y el Hombre, 25(3) https://www.uv.mx/cienciahombre/revistae/vol25num3/articulos/efectos-luz/

Sánchez, G. \& Nova, J. (2008). Confiabilidad y reproducibilidad de la escala de fototipos de Fitzpatrick antes y después de un ejercicio de estandarización clínica. Biomédica, 28(4), 544. Doi: https://doi.org/10.7705/biomedica.v28i4.59

Sánchez, G., Nova, J., Rodriguez-Hernandez, A., Solorzano-Restrepo, C., Gonzalez, J. \& Olmos, M. (2014). Sun protection for preventing basal cell and squamous cell skin cancers. Cochrane Database Of Systematic Reviews. CD011161http://dx.doi.org/10.1002/14651858. cd011161

Tania, F., Bruna, B., Aline, R. (2008). Dermatología pediátrica Latinoamericana, Revista Oficial de la Sociedad Latinoamericana de Dermatología Pediátrica. Fotoprotección en la infancia, 6(1), 40-45, doi: http://docplayer.es/18257082-Dermatologia-pediatrica-latinoamericana-revista-oficial-de-la-sociedad-latinoamericana-de-dermatologia-pediatrica.html

Valdivielso-Ramos, M. \& Herranz, J. (2010). Actualización en fotoprotección infantil. Anales de Pediatría, 72(4), 282.e1-282.e9, doi: http://dx.doi.org/10.1016/j.anpedi.2009.06.013 